\title{
El Propóleos Reduce la Esteatosis Hepática Inducida por Dieta en Ratones
}

\author{
Propolis Decrease Diet-Induced Hepatic Steatosis in Mice
}

\author{
*Christian L. Herrera; ***Osvaldo Fritz; *** Gloria Montenegro; \\ *****Marysol Alvear; ${ }^{* * * * * *}$ Mariano del Sol \& ${ }^{* * * * * *}$ Luis A. Salazar
}

HERRERA, C. L.; FRITZ, O.; MONTENEGRO, G., ALVEAR, M.; DEL SOL, M. \& SALAZAR, L. A. El propóleos reduce la esteatosis hepática inducida por dieta en ratones. Int. J. Morphol., 28(1):75-84, 2010.

RESUMEN: El hígado graso no alcohólico (HGNA), comprende un amplio espectro de lesiones, que van desde esteatosis hepática, hasta cirrosis y carcinoma hepatocelular, siendo sus principales factores de riesgo los desordenes asociados a síndrome metabólico (SM). El propóleos, sustancias resinosa elaborada por Apis mellifera para la protección de la colmena, ha demostrado un efecto hepatoprotector, así el objetivo de esta investigación fue evaluar el efecto de un propóleos chileno sobre el desarrollo de esteatosis hepática no alcohólica en ratones C57BL/6J sometidos a una dieta aterogénica tipo Paigen. Veintiocho ratones (C57BL/6J), divididos en: 1, dieta balanceada $(\mathrm{CH}) ; 2$, dieta hipercolesterolémica $(\mathrm{HC}) ; 3$, dieta $\mathrm{HC}+10 \mathrm{mg} / \mathrm{kg} /$ día de propóleos (BP); 4, dieta HC + 40 mg/kg/día de propóleos (AP). Después de 16 semanas, se determinó la concentración de glucosa, colesterol total, triglicéridos y los niveles de alanina aminotransferasa (ALAT). El tejido hepático fue fijado en una solución de formalina tamponada al 10\% para, posteriormente, ser incluido en paraplast y teñido con Hematoxilina-Eosina y fragmanetos congelados a $-30^{\circ} \mathrm{C}$ fijados en formalina teñidos con tinción Oil red O. No existieron diferencias en la concentración de glucosa ni triglicéridos, a diferencia del colesterol total, entre el grupo alimentado con dieta balanceada (CH) y los alimentados con la dieta aterogénica D12336 (HC, BP y AP). De la misma forma se observó que existen diferencias en los niveles de actividad de ALAT entre los grupos estudiados, destacando su reducción en los grupos suplementados con propóleos (BP y AP). De forma concordante, la histoarquitectura del grupo HC, mostró esteatosis simple y focos de infiltrado leucocitario en el lobulillo hepático, observándose una disminución gradual de las alteraciones en los grupos de BP y AP. En conclusión, el propóleos chileno estudiado disminuye la esteatosis hepática inducida por la dieta aterogénica tipo Paigen en ratones C57BL/6J, sin embargo, los mecanismos implicados en esta actividad hepatoprotectora tienen que ser identificados.

PALABRAS CLAVE: Esteatosis hepática; HGNA; Dieta aterogénica; Propóleos.

\section{INTRODUCCIÓN}

Por sus propiedades benéficas para la salud humana, el propóleos ha sido utilizado por la medicina tradicional desde hace siglos, sin embargo, estas características han sido explicadas científicamente sólo en los últimos años. Esta sustancia resinosa, elaborada por Apis mellifera para la protección de la colmena, ha demostrado diversas propiedades, tales como: antiinflamatorio, antitumoral, antioxidante y antimicrobiano (Burdock, 1998; Banskota et al., 2001; Sforcin, 2007; Nakajima et al., 2009; Herrera et al., 2010); no obstante, es muy importante tener presente que la composición de los propóleos y su actividad biológica, muestran una importante variabilidad cualitativa y cuantitativa (Kujumgiev et al., 1999; Velikova et al., 2000), y que sus características dependen del origen botánico y geográfico

\footnotetext{
Estudiante Programa de Doctorado en Ciencias, mención Biología Celular y Molecular Aplicada; Depto. de Ciencias Preclínicas, Facultad de Medicina, Universidad de La Frontera, Temuco, Chile.

** Estudiante Programa de Magister en Ciencias, mención Morfología, Universidad de La Frontera, Temuco, Chile; Facultad Ciencias de la Salud, Universidad San Sebastián, Concepción, Chile.

*** Profesora Titular, Depto. de Ciencias Vegetales, Facultad de Agronomía e Ingeniería Forestal, Pontificia Universidad Católica de Chile, Santiago, Chile.

**** Profesora Asociado, Depto. de Ciencias Químicas, Facultad de Ingeniería, Ciencias y Administración, Universidad de La Frontera, Temuco, Chile.

***** Unidad de Anatomía, Depto. de Ciencias Básicas, Facultad de Medicina, Universidad de La Frontera, Temuco, Chile.

${ }^{* * * * * * *}$ Laboratorio de Biología Molecular \& Farmacogenética, Departamento de Ciencias Básicas, Facultad de Medicina; Núcleo de Desarrollo CientíficoTecnológico en Biorecursos, Universidad de La Frontera, Temuco, Chile. Este trabajo fue financiado mediante Proyecto FONDEF de CONICYT N ${ }^{\circ}$ D05I10021
} 
de este producto (Bankova et al., 2000; Hegazi et al., 2000; Marcucci et al., 2000; Peña, 2008).

El hígado graso no alcohólico (HGNA) es la enfermedad hepática más común en los países occidentales, comprende un amplio espectro de lesiones, que van desde esteatosis hepática, hasta cirrosis y carcinoma hepatocelular; siendo sus principales factores de riesgo los desordenes asociados a síndrome metabólico (SM) como: obesidad, resistencia a la insulina, intolerancia a la glucosa y dislipidemia, por lo que HGNA se puede considerar como una manifestación hepática del SM (Machado \& Cortez-Pinto, 2006; Yan et al., 2007; Schreuder et al., 2008).

La dieta aterogénica tipo Paigen (15\% grasa, 1.25\% colesterol y $0.5 \%$ ácido cólico) ha sido utilizada en animales para la evaluación de los trastornos provocados por niveles elevados de colesterol, tales como aterosclerosis, litiasis hepática, alteraciones microvasculares (Paigen et al., 1987; Wittenburg et al., 2003; Getz \& Reardon, 2006; Stokes, 2006). Además, es importante mencionar que el estudio del efecto de los componentes de la dieta Paigen sobre el tejido hepático reveló que el colesterol induce la expresión de genes relacionados al proceso inflamatorio y que el acido cólico activa la expresión de genes relacionados a fibrosis hepática (Vergnes et al., 2003), ambos procesos observados en HGNA.

La actividad hepatoprotectora del propóleos ha sido evaluada previamente en modelos animales donde se indujo el daño mediante la exposición a diferentes sustancias, como son el alcohol, tamoxifeno, acetaminofeno, tetracloruro de carbono (CCl4), diethylnitrosamina y hexachlorobenzeno (Giurgea et al., 1987; Merino et al., 1996; Seo et al., 2003; Chen et al., 2008; Nirala \& Bhadauria, 2008; Albukhari et al., 2009; Sathiavelu et al., 2009). Los resultados observados en general son positivos, demostrando en la mayoría de los casos un efecto hepatoprotector.

Los mecanismos que sustentan la actividad hepatoprotectora hasta la fecha, serian explicados por su composición de minerales, flavonoides y ácidos fenólicos, los cuales por ejemplo, en el caso del daño por alcohol, ejercerían su acción al disminuir la expresión de genes asociados a inflamación y fibrogénesis (Chen et al.) y en el caso del daño por $\mathrm{CCl} 4$, reduce los niveles de enzimas hepáticas, urea y acido úrico; mejora la actividad de enzimas metabolizadoras de drogas, inhibe la peroxidación lipídica y favorece la actividad de glutatión (Bhadauria et al., 2008).

Considerando estos antecedentes, el objetivo de esta investigación fue evaluar el efecto de propóleos chilenos sobre el desarrollo de esteatosis hepática no alcohólica en ratones $\mathrm{C} 57 \mathrm{BL} / 6 \mathrm{~J}$ sometidos a una dieta aterogénica.

\section{MATERIAL Y MÉTODO}

Preparación y Análisis del Extracto de Propóleos. El propóleos utilizado en este ensayo fue recolectado en la zona cordillerana de la Región de La Araucanía, Chile (-38.930408 Latitud, -72.026752 Longitud), localidad caracterizada por un clima templado lluvioso con influencia mediterránea. Para la determinación del origen botánico, se procedió a realizar el análisis palinológico (análisis de restos vegetales, principalmente polen) de la siguiente forma: 30 gramos de propóleos crudo fueron disueltos en etanol al 70\% hasta completar $100 \mathrm{ml}$ de mezcla. Luego, de esta solución fue extraído el sobrenadante, el que fue utilizado para la suplementación del alimento, y el residuo sólido generado fue analizado mediante microscopía óptica, realizándose la identificación de las estructuras presentes en la muestra.

Además, se determinó la composición química del propóleos mediante un Cromatógrafo Líquido de Alta Precisión (HPLC) Merck-Hitachi, equipado con una bomba modelo L-6200, un detector UV-visible, modelo L-4200 y un horno para columna Phenomenex Terma Sphere, modelo TS-130. La separación se realizó en una columna RP-18 (12,5 x 0,4 cm, tamaño de partícula $5 \mu \mathrm{m}$ ) (Merck, Alemania), la cual se eluyó a $25^{\circ} \mathrm{C}$ utilizando como fase móvil la mezcla de ácido fórmico $5 \%$ en agua (A) y metanol (B). La separación de los compuestos se realizó mediante una corrida isocrática de 0 a 10 minutos, con la mezcla A $70 \%$ y B $30 \%$, seguida de un gradiente hasta $100 \% \mathrm{~B}$ a los 70 minutos. Los compuestos se detectaron a una longitud de onda de $290 \mathrm{~nm}$, con una sensibilidad de 0,001; el volumen de inyección fue $10 \mu \mathrm{L}$. La identificación de los compuestos fenólicos se realizó mediante el uso de los estándares miricetina, kaempferol, quercetina, ácido cafeico, galangina, pinocembrina, apigenina, ácido cafeico fenil éster (CAPE) y resveratrol (Sigma, EE.UU.).

Animales y Diseño Experimental. Se utilizaron 28 ratones C57BL/6J, obtenidos del Bioterio Central de la Universidad de Chile (Santiago, Chile), fueron divididos en cuatro grupos de 7 individuos, siendo sometidos a un periodo de adaptación de 2 semanas con ciclos de luz oscuridad de 12 horas y temperatura entre 20 a $23{ }^{\circ} \mathrm{C}$ con libre acceso a alimentación balanceada (Champion S. A., Santiago, Chile). Luego, a partir de la decima semana y hasta la vigésimo sexta semana de vida, los animales de los grupos 2 (hipercolesterolémicos, HC), 3 y 4 fueron alimentados con dieta aterogénica (Dieta tipo Paigen, D12336, Laboratorios ResearchDiets, New Jersey, USA).

Además, de la dieta aterogénica a los grupos 3 y 4 , se les suplementó el alimento con $10 \mathrm{mg} / \mathrm{Kg}$ /día (bajo propóleos, BP) y $40 \mathrm{mg} / \mathrm{Kg} /$ día (alto propóleos, AP) de la fracción soluble en etanol, la cual fue previamente liofilizada. El manejo de los 
animales fue acorde con lo señalado en el Manual de Normas de Bioseguridad del Comité Nacional de Biotecnología de CONICYT, publicado en el año 2008. Además, todos los experimentos realizados fueron aprobados por el Comité de Ética institucional.

Determinaciones Bioquímicas. Después de 12 horas de ayuno, se obtuvieron mediante punción cardíaca, muestras de sangre las cuales fueron centrifugadas a $10000 \mathrm{~g}$ por $10 \mathrm{~min}$. El suero obtenido fue almacenado a $-20^{\circ} \mathrm{C}$ hasta que se realizaron las determinaciones de glucosa, colesterol total y triglicéridos por métodos enzimáticos-colorimétricos (Barham \& Trinder, 1972; Fossati \& Medicci, 1987; Fossati \& Prencipe, 1982) y los niveles de Alanina aminotransferasa (ALAT) mediante un ensayo cinético-enzimático (Bergmeyer et al., 1978). Todas las determinaciones fueron realizadas en un fotómetro semiautomático Humalyzer 3000 (Human Diagnostics, Alemania).

Análisis Histológico. El tejido hepático fue fijado en una solución formalina neutra tamponada al $10 \%$, para posteriormente ser incluido en parafina y teñido con Hematoxilina-Eosina (Merck, Alemania). Los cortes fueron realizados de forma seriada en micrótomo Leica RT 2125 con $4 \mu \mathrm{m}$ de espesor. Posteriormente, se efectuó un análisis de la esteatosis hepática, siendo clasificada de forma gradual de la siguiente forma: 0 , ausente; $1,1-25 \%$; 2, 26-50\%; 3, 51-75\%; 4, 76-100\% de los hepatocitos (Kleiner et al., 2005).

Además, fragmentos congelados a $-30^{\circ} \mathrm{C}$ de hígados fijados en formalina, fueron teñidos con tinción Oil red O (Merck, Alemania), con el objetivo de demostrar la presencia de lípidos.
Análisis estadístico. Los resultados fueros expresados como promedios, desviaciones estándar, valores máximos y mínimos. El análisis de los datos obtenidos se realizó utilizando el Programa GraphPad Prism, versión 5.0 (USA). Para las comparaciones de los datos cuantitativos entre los grupos se utilizo ANDEVA. El nivel de significancia estadística considerado fue $\mathrm{P}<0.05$.

\section{RESULTADOS}

En la muestra de propóleos estudiada se pudo observar la presencia de 19 especies vegetales, entre las cuales predominó la especie Lotus uliginosus, herbácea introducida en Chile, muy frecuente entre la sexta y la undécima región. A pesar del predominio de esta especie foránea, el resto de las estructuras observadas correspondió a especies provenientes de árboles y arbustos nativos (Fig. 1). El análisis cromatográfico, en las condiciones ya señaladas, indica que el propóleos utilizado presenta concentraciones de ácido cafeico, miricetina, quercetina, kaempferol, apigenina, pinocembrina, galangina y CAPE (Fig. 2).

En la Fig. 3 se puede observar que no existen diferencias significativas en los niveles de glucosa ni triglicéridos, a diferencia de lo ocurrido con la concentración de colesterol total, donde se observó diferencias entre el grupo alimentado con dieta balanceada $(\mathrm{CH})$ y los alimentados con la dieta aterogénica para roedores D12336 (HC, BP y AP). De la misma forma se pudo observar que existen diferencias significativas en los niveles de actividad de ALAT entre los grupos estudiados, destacando su reducción en los grupos suplementados con propóleos (Fig. 4).

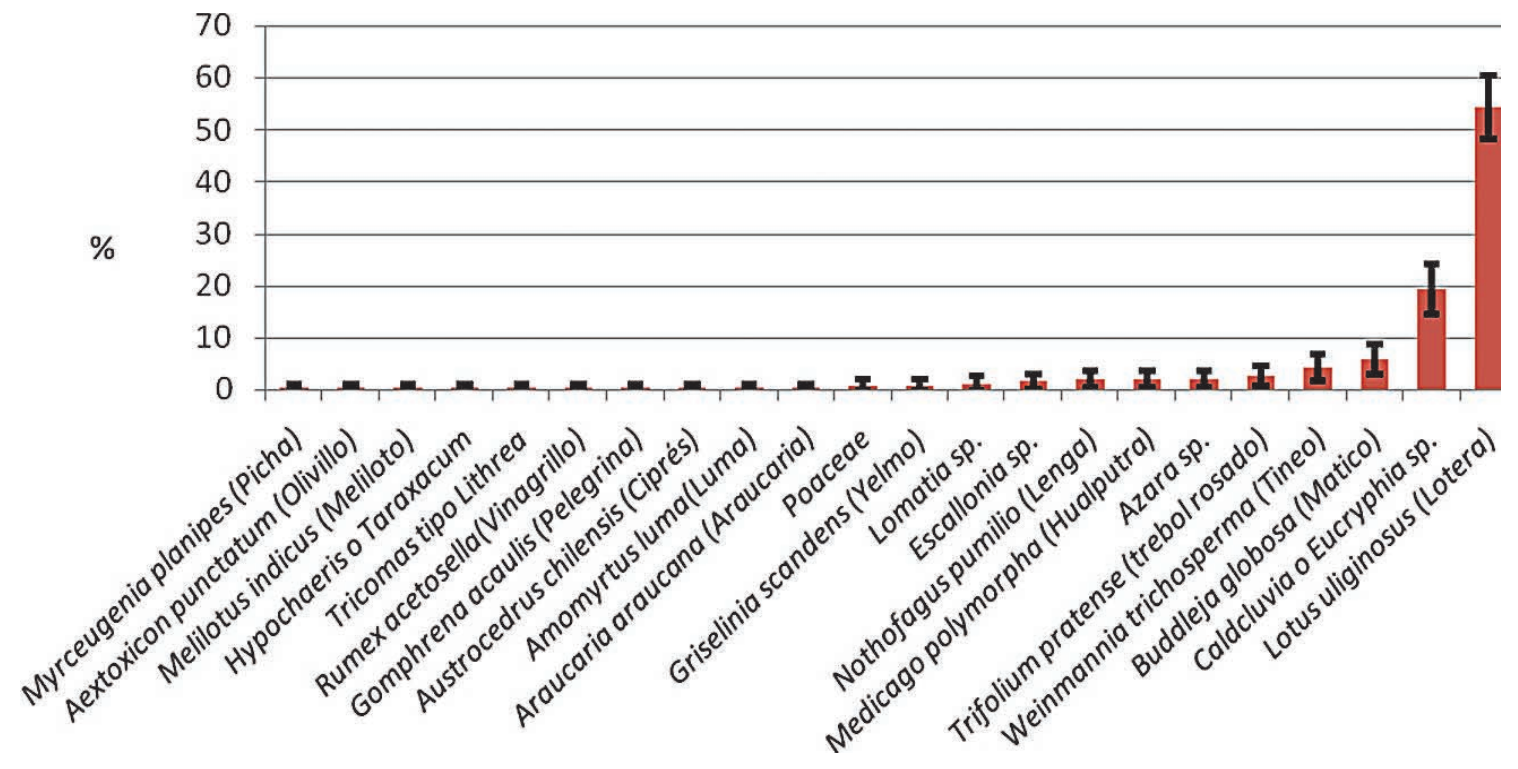

Fig. 1. Caracterización botánica del propóleos chileno utilizado. Frecuencia (\%) de gramos de polen observada. 
HERRERA, C. L.; FRITZ, O.; MONTENEGRO, G., ALVEAR, M.; DEL SOL, M. \& SALAZAR, L. A. El propóleos reduce la esteatosis hepática inducida por dieta en ratones. Int. J. Morphol., 28(1):75-84, 2010.

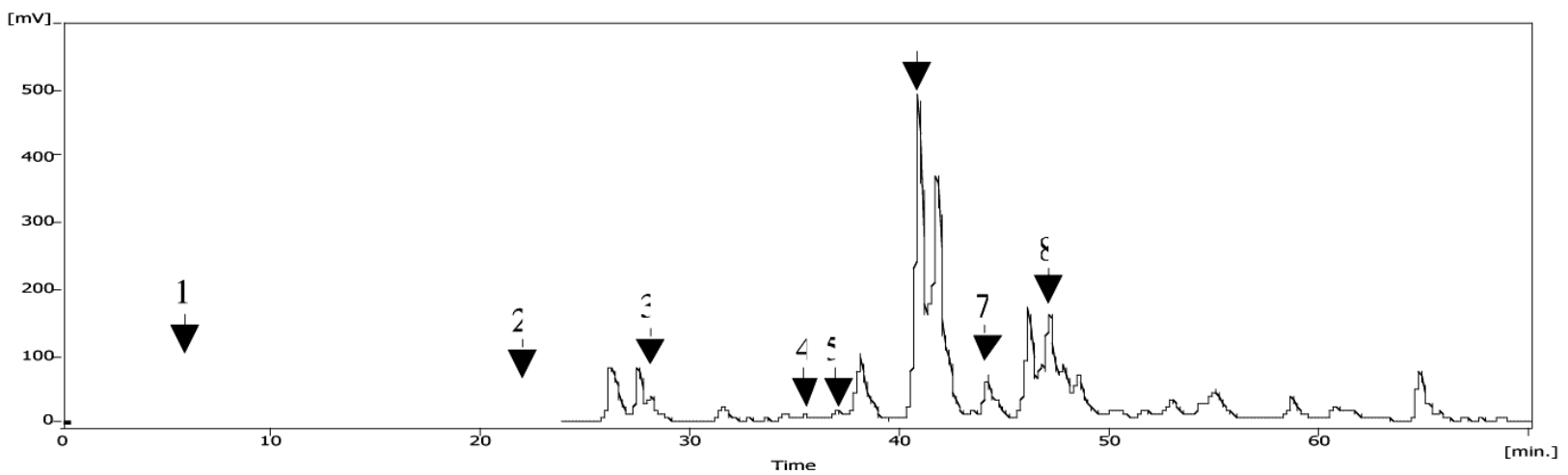

Fig. 2. Análisis cromatográfico del propóleos chileno utilizado en este estudio. Longitud de onda: 290 nm, volumen de inyección: $10 \mu \mathrm{L}$. Compuestos identificados: 1, ácido cafeico; 2, miricetina; 3, quercetina 4, kaempferol; 5, apigenina; 6, pinocembrina; 7, galangina; 8, ácido cafeico fenil éster (CAPE).
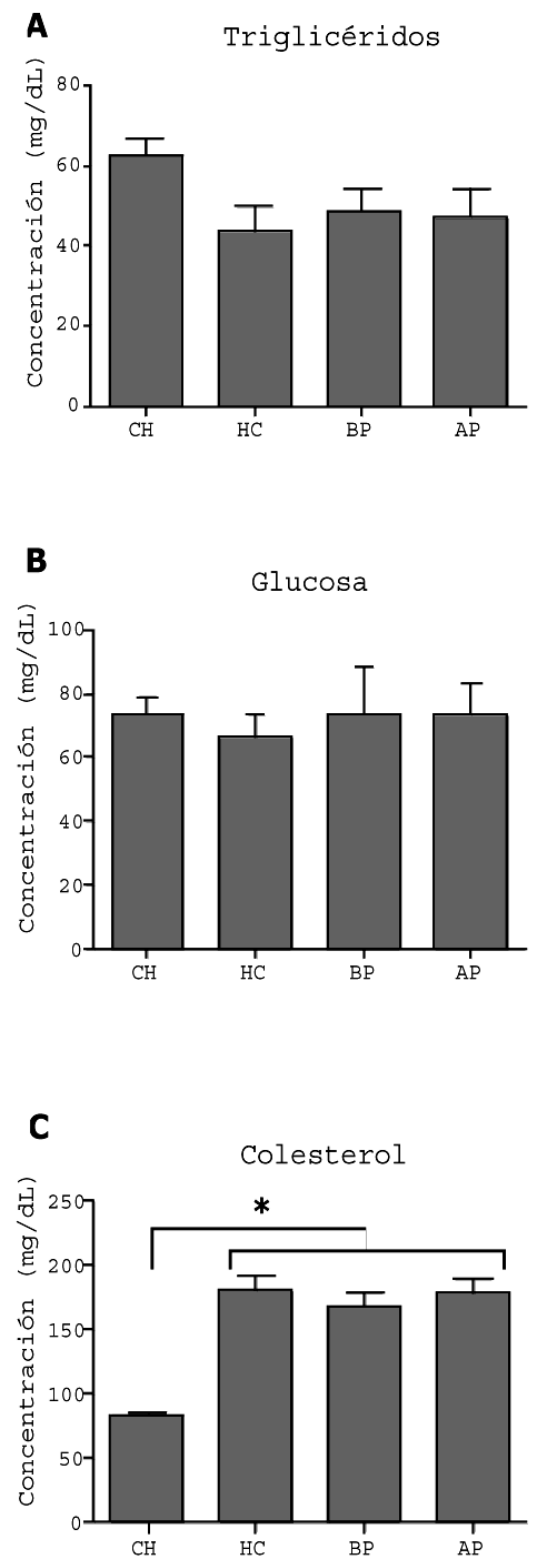

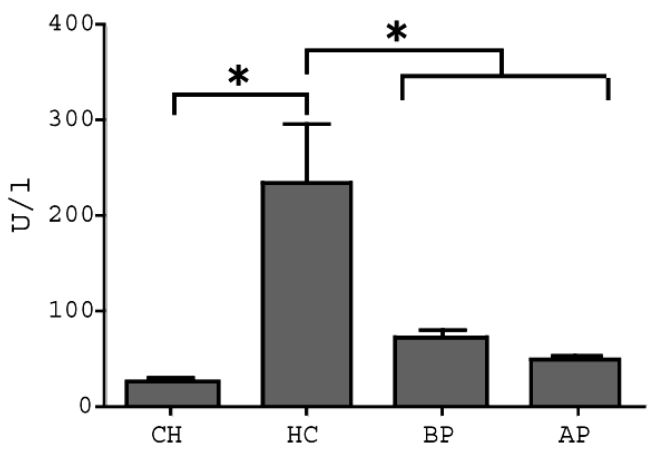

Fig. 4. Niveles de actividad sérica de Alanina aminotransferasa (ALAT) en los diferentes grupos de estudio. Se observó diferencia entre los animales alimentados con dieta tradicional para roedores versus dieta aterogénica $(\mathrm{p}<0.05)$ y reducción significativa de los niveles de actividad de la enzima en los grupos suplementados con propóleos al compararlos con el grupo sin suplementación de propóleos $(\mathrm{p}<0.05)$. Los valores corresponden al promedio $\pm \mathrm{DE}$ de 5 a 7 animales por grupo. $\mathrm{CH}$, Dieta balanceada; HC, Dieta aterogénica D12336; BP, Dieta baja en propóleos (10 mg/ $\mathrm{Kg}$ /día); AP, Dieta alta en propóleos (40 mg/Kg/día).

Fig. 3. Concentraciones séricas de triglicéridos (A), glucosa (B) y colesterol total (C) en los diferentes grupos de ratones estudiados. Sólo se observaron diferencias en la concentración de colesterol total entre los animales alimentados con dieta aterogénica versus dieta tradicional para roedores $(\mathrm{p}<0.0001)$. Los valores corresponden al promedio $\pm \mathrm{DE}$ de 5 a 7 animales por grupo. $\mathrm{CH}$, Dieta balanceada; $\mathrm{HC}$, Dieta aterogénica D12336; BP, Dieta baja en propóleos (10 mg/Kg/día); AP, Dieta alta en propóleos (40 $\mathrm{mg} / \mathrm{Kg} / \mathrm{día})$. 


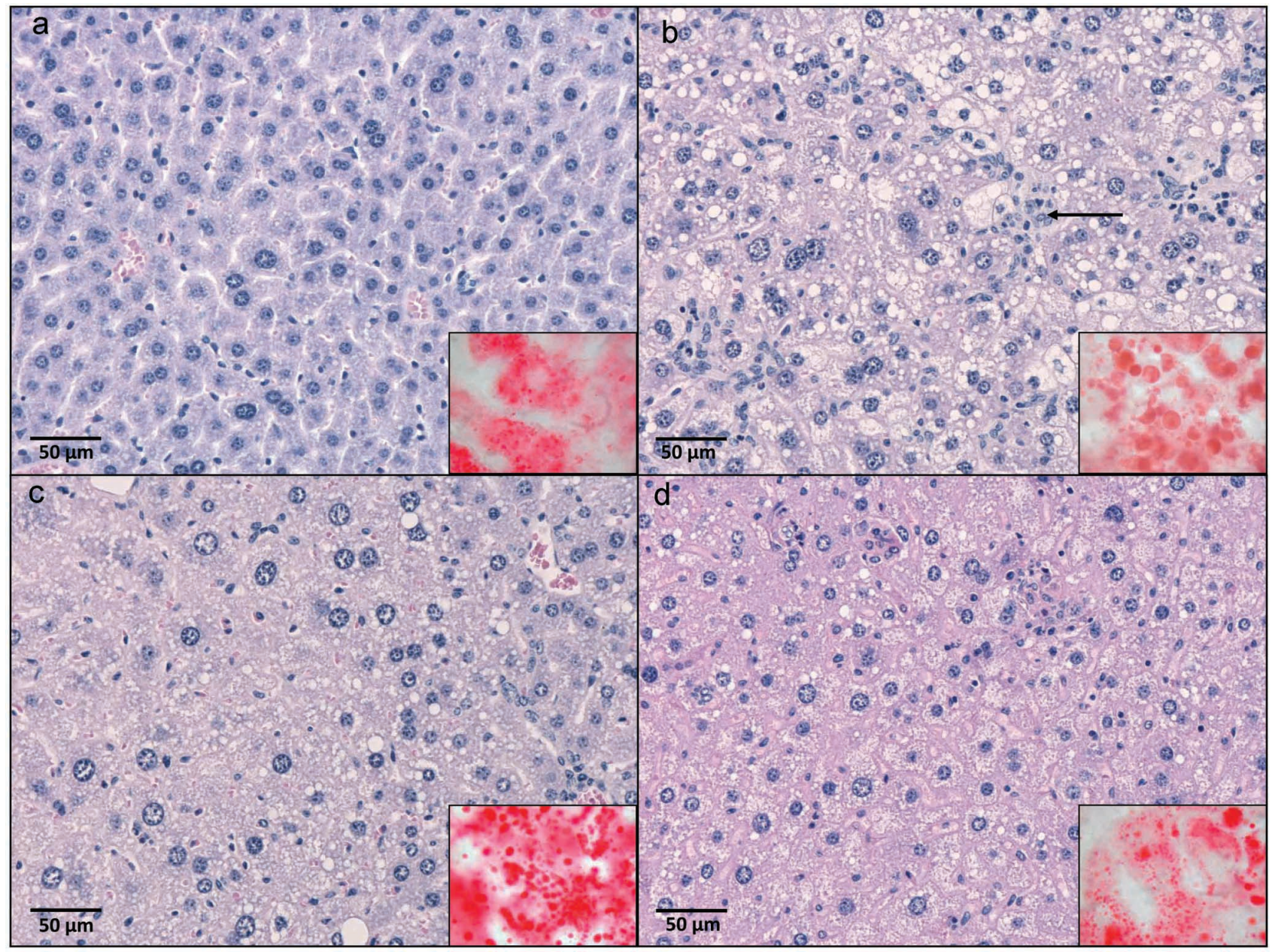

Fig. 5. Cortes histológicos representativos de la morfología hepática observada en los grupos estudiados. Tinción H\&E; cuadro inferior derecho: Tinción Oil red O, aumento 1000X. a.- $\mathrm{CH}$, dieta balanceada; b.- HC, dieta aterogénica; c.- $\mathrm{BP}$, dieta baja en propóleos (10 mg/Kg/ día); d.- AP, dieta alta en propóleos (40 mg/Kg/día). Los hígados de los animales alimentados con dieta balanceada se observan normales. Los animales del grupo HC muestran citoplasma espumoso y presencia de vacuolas grasas gigantes. Se observa además la presencia de infiltrado inflamatorio (flecha). Los grupos con suplementación de propóleos muestran una disminución de estas características.

El análisis morfológico de los hígados de los animales tratados con la dieta aterogénica mostró esteatosis simple centrolobulillar y panlobulillar, se presentaron células con citoplasma espumoso y presencia de vacuolas grasas gigantes que desplazan al núcleo del hepatocito hacia la periferia, en algunas áreas, los sinusoides se presentaban congestionados y las células fagocitarias hepáticas hiperplásicas. La vena centrolobulillar se mostró dilatada y congestionada, sin necrosis focal o ruptura. Sólo se observaron peque- ños focos de infiltrado leucocitario en el lobulillo hepático. Los espacios porta se mostraban levemente desorganizados. El volumen relativo de los conductos biliares y vasos sanguíneos, así como la densidad de superficie de las arterias presentaron alteraciones leves a moderadas. Estas características morfológicas mostraron una disminución gradual de las alteraciones en los grupos de animales sometidos a suplementación con propóleos en baja y alta concentración, respectivamente (Tabla I y Fig. 5).

Tabla I. Efecto del consumo de propóleos sobre el grado de esteatosis hepática en ratones sometidos a una dieta aterogénica durante 16 semanas.

\begin{tabular}{lcccc}
\hline & \multicolumn{4}{c}{ Grupos } \\
& $\mathrm{CH}$ & $\mathrm{HC}$ & $\mathrm{BP}$ & $\mathrm{AP}$ \\
\hline Grado Esteatosis & $0,0 \pm 0,0$ & $3,2 \pm 0,6$ & $2,4 \pm 0,5^{*}$ & $2,4 \pm 0,6^{*}$ \\
\hline
\end{tabular}

Los valores corresponden al promedio \pm DE de 5 a 7 animales por grupo. $\mathrm{CH}$, Dieta balanceada; HC, Dieta aterogénica D12336; BP, Dieta baja en propóleos (10 mg/Kg/día); AP, Dieta alta en propóleos (40 mg/Kg/día). $* \mathrm{P}<0,001$ comparado con el grupo HC. 


\section{DISCUSIÓN}

A pesar de que todos los propóleos muestran algún grado de actividad biológica, es importante destacar la determinación del origen botánico-geográfico del propóleos estudiado, debido a que se encuentra bien documentado que existe variaciones en el grado de actividad de los productos apícolas en función de este factor (Bankova et al.). También es relevante hacer mención que el valor de la caracterización, botánica y/o química, va más allá de la importancia académica, ya que puede generar valor agregado a la producción apícola.

En nuestro estudio, la especie encontrada con mayor frecuencia fue $L$. uliginosus (herbácea introducida), al igual que en las muestras de propóleos de la zona central, destaca la ausencia de especies introducidas que son grandes productoras de resinas, tales como los árboles forestales de los géneros Eucalyptus y Pinus, frecuentes en la zona y presentes en todas las muestras de propóleos estudiadas en la zona central de Chile (Montenegro et al., 2004), esta situación es importante, ya que podría indicar que las abejas preferirían buscar recursos para la elaboración de propóleos en árboles $\mathrm{y}$ arbustos nativos.

Previamente han sido evaluados los efectos de extractos acuosos y etanólicos de propóleos y algunos de sus compuestos, sobre el hígado y metabolismo lipídico de ratas y ratones. De esta forma, se ha podido demostrar el efecto hepatoprotector de propóleos de origen ruso, cubano, chino, koreano, indio, argentino, alemán, turco y brasileño y argentino. En este sentido y a modo de ejemplo, podemos señalar que el propóleos de origen indio, brasileño y cubano lograron disminuir el daño provocado por la exposición a $\mathrm{CCl} 4$, atribuyéndose principalmente esta actividad a su composición de minerales, flavonoides, compuestos polifenólicos y a las propiedades antioxidantes de estos últimos (Basnet et al., 1996; Merino et al.; Bhadauria et al., 2007; Bhadauria et al., 2008). De la misma forma investigadores indios demostraron que la ingesta de propóleos tiene la capacidad de mejorar la histoarquitectura hepatorenal, parámetros bioquímicos y la capacidad antioxidante en animales expuestos a acetaminofeno, imputando estos efectos al poder antioxidante de los compuestos fenólicos presentes (Nirala $\&$ Baddauria). Sumado a estos antecedentes, un propóleos de origen coreano demostró la capacidad de disminuir el daño parenquimatoso inducido por acetaminofeno, en ratas y ratones pre-tratados con diferentes dosis de propóleos; sin embargo, los investigadores demuestran que el efecto hepatoprotector no sólo se debería a la acción antioxidante, si no que sería resultado de la disminución de la generación de $\mathrm{N}$-acetyl-p-benzoquinoneimina, metabolito altamente reactivo responsable de la toxicidad de acetaminofeno, por la vía de citocromo P450, a su vez que aumentaría la detoxificación al promover la actividad de enzimas encargadas de la conjugación de productos para su eliminación (Seo et al.). Asimismo, un extracto etanólico de propóleos de origen chino, ha demostrado la capacidad de disminuir la extensión del hígado graso alcohólico al actuar previniendo la elevación de enzimas microsomales y el aumento de la peroxidación de lípidos. Observándose, además, un aumento de la actividad de glutatión-S-transferasa y gamaglutamilcisteina sintetasa y la disminución de los niveles de glutatión en tejido hepático (Lin et al., 1997; Lin et al., 1999). Finalmente, en el caso de propóleos de origen argentino se demostró la capacidad de disminuir la extensión de lesiones cirróticas causadas por tioacetamida en ratas (Chen et al.).

Chen et al. también demostraron que la pinocembrina, compuesto polifenólico presente en el propóleos, podría prevenir el daño tisular, ya que se observó que en cultivos de células Hep 3B se logra inhibir la expresión de la transglutaminasa tisular (tTG), enzima relacionada con daño hepático agudo y fibrosis, mediada probablemente por la inactivación de las vías ERK1/2 y PI3K/Akt; regulando de esta forma la transcripción génica dependiente de NFkappaB, factor de transcripción que activa la expresión de genes involucrados en respuestas inflamatorias, inmunes y de fase aguda. Otros compuestos polifenólicos del propóleos que se han evaluado son: la crisina, presente también en plantas y mieles de abeja, que demostró ofrecer protección contra el estrés oxidativo en ratas expuestas a ingesta excesiva de alcohol (Sathiavelu et al.), y el ácido cafeico fenil éster (CAPE), el cual evidenció actividad hepatoprotectora al ser administrado a ratones expuestos a tamoxifeno (Albukhari et al.).

A la hora de comparar nuestros resultados, podemos señalar que un estudio efectuado con el objetivo de evaluar el efecto de propóleos brasileños sobre el metabolismo lipídico de ratas, se determinó que utilizando una dieta alta en grasas (20\%) suplementada con diferentes concentraciones de propóleos $(0,5$ y $0,05 \%)$, logra en dosis alta, una disminución significativa tanto de colesterol total como de triglicéridos, señalando además, que su administración posiblemente mejore la acumulación de grasa corporal y la dislipidemia a través de cambios en la expresión de genes relacionados al depósito de tejido adiposo y metabolismo lipídico (Ichi et al., 2009). De la misma forma, investigadores japoneses determinaron un efecto similar al descrito previamente, utilizando también una dieta alta en grasa en ra- 
tones C57BL/6 y una dosis de $10 \mathrm{mg} / \mathrm{kg} /$ día durante 10 días, sin embargo, al evaluar durante 4 semanas en ratones obesos dosis de de 5 y $50 \mathrm{mg} / \mathrm{kg} /$ día solo observaron una disminución en los niveles de glucosa, no observando diferencias en colesterol total ni triglicéridos (Koya-Miyata et al., 2009). Este último antecedente resulta relevante, ya que la concentración que no logró disminuir los lípidos sanguíneos es similar a la utilizada en nuestro ensayo, por lo que la concentración de propóleos, además del diferente origen y composición de los propóleos, podría explicar el que en nuestra investigación no se hayan encontrado diferencias en el perfil lipídico.

La dieta Paigen tiene la capacidad de inducir hipercolesterolemia, producto que el acido cólico acentúa el efecto de la dieta alta en colesterol y grasas, ya que facilita la absorción de colesterol e inhibiría la actividad de 7 alfa-hidroxilasa de colesterol (CYP7A1), enzima clave en la síntesis de ácidos biliares, induciendo además la acumulación de lípidos, inflamación y fibrosis hepática en roedores (Ando et al., 2005). Siendo esta la dieta utilizada en nuestra investigación podemos señalar que de forma similar a lo observado por otros investigadores (Matsuzawa et al., 2007; Desai et al., 2008), en nuestro ensayo se observó infiltrado leucocitario, la presencia de vacuolas lipídica en los hepatocitos, aumento de ALAT e hipercolesterolemia en los animales expuestos a la dieta aterogénica. A pesar de esto, es importante mencionar que si bien con este modelo, se logra desarrollar HGNA, no se logra representar por completo el contexto metabólico de la patología (obesidad, resistencia a la insulina, intolerancia a la glucosa y síndrome metabólico), por lo que presenta limitaciones para la investigación de la patogenia o de intervenciones terapéuticas (Larter \& Yeh, 2008).
En el contexto de la protección sobre el desarrollo de esteatosis inducida por una dieta aterogénica, de acuerdo a nuestra información, no existen antecedentes sobre el uso de propóleos, motivo por el que resulta relevante destacar nuestros resultados. Sólo se pueden encontrar referencias respecto de los efectos del resveratrol, principal efector de las propiedades cardioprotectoras del vino tinto, compuesto que también se encuentra presente en propóleos y en una gran variedad de vegetales (Ahn et al., 2008; Bujanda et al., 2008; Shang et al., 2008). Así, Ahn et al. demostraron, en ratones, que la adición de resveratrol (aproximadamente $20 \mathrm{mg} / \mathrm{kg} / \mathrm{día}$ ) protege contra el desarrollo de sobrepeso, hepatomegalia, dislipidemia y esteatohepatitis inducida por dieta aterogénica, explicando que este compuesto suprime la sobreexpresión de genes relacionados a la lipogénesis y estimula la expresión de genes relacionados con la lipólisis. Tanto resveratrol, como los compuestos CAPE y especialmente, pinocembrina, se encuentran en la muestra de propóleos estudiada, por lo que al menos, parcialmente pudiéramos atribuir el efecto observado a su acción. No obstante, es importante tener presente que los resultados obtenidos en animales no siempre son extrapolables a humanos y que en concordancia con esta aseveración, como fue descrito, muchos de los efectos del propóleos son atribuidos a sus efectos antioxidantes, sin embargo, en humanos la actividad hepatoprotectora de suplementos alimenticios con actividad antioxidante, en pacientes con HGNA, no es posible afirmarla ni rechazarla debido a que la evidencia actual no es concluyente (Flavio et al., 2007).

En conclusión, el propóleos chileno estudiado disminuye la esteatosis hepática inducida por la dieta Paigen en ratones C57BL/6J, sin embargo, los mecanismos implicados en esta actividad hepatoprotectora tienen que ser identificados.

HerRerA, C. L.; FRITZ, O.; MONTENEgro, G., ALVEAR, M.; DEL SOL, M. \& SALAZAR, L. A. Propolis decrease dietinduced hepatic steatosis in mice. Int. J. Morphol., 28(1):75-84, 2010.

SUMMARY: Nonalcoholic fatty liver disease (NAFLD) covers a wide spectrum of injuries ranging from simple steatosis to cirrhosis and hepatocellular carcinoma. Its main risk factors are disorders associated with metabolic syndrome (MS). Propolis, a resinous substance produced by Apis mellifera to protect is hive, has demonstrated a hepatoprotective effect. Thus, the aim of this study was to evaluate the effect of Chilean propolis on development of nonalcoholic hepatic steatosis in C57BL/6J mice exposed to Paigen atherogenic diet. Twenty eight mice C57BL/6J were divided four groups: 1, balanced diet (CH); 2, hypercholesterolemic diet (HD); 3 , HD diet supplemented with $10 \mathrm{mg} / \mathrm{kg} /$ day of propolis (LP); 4, HD diet supplemented with $40 \mathrm{mg} / \mathrm{kg} /$ day of propolis (HP). After 16 weeks of treatment was determined glucose, total cholesterol and triglycerides concentrations and alanine aminotransferase (ALT) activity. The liver tissue was fixed in $10 \%$ buffered formalin solution, embedded in paraplast and stained with hematoxylin-eosin. No differences was detected in glucose and triglycerides concentrations, contrasting with total cholesterol levels between group fed with a balanced diet $(\mathrm{CH})$ and feed with atherogenic diet D12336 (HD, LP and HP). In the same way, it was noted differences in ALT activity between groups, standing out the reduction in propolis supplemented groups (LP and HP). Likewise the histoarchitecture of HD group, showed simple steatosis, inflammatory cell infiltration and inflammatory foci in hepatic lobule. This characteristics show a gradual decrease in LP and HP groups. In conclusion, the Chilean propolis analyzed decreases hepatic steatosis induced by atherogenic diet in C57BL/6J mice. However, the involved mechanisms in this hepatoprotective activity must be identified. 


\section{REFERENCIAS BIBLIOGRÁFICAS}

Ahn, J.; Cho, I.; Kim, S.; Kwon, D. \& Ha, T. Dietary resveratrol alters lipid metabolism-related gene expression of mice on an atherogenic diet. J. Hepatol., 49:1019-28, 2008.

Albukhari, A. A.; Gashlan, H. M.; El-Beshbishy, H. A.; Nagy, A. A. \& Abdel-Naim, A. B. Caffeic acid phenethyl ester protects against tamoxifen-induced hepatotoxicity in rats. Food Chem. Toxicol., 47:168995, 2009.

Ando, H.; Tsuruoka, S.; Yamamoto, H.; Takamura, T.; Kaneko, S. \& Fujimura, A. Regulation of cholesterol 7alpha-hydroxylase mRNA expression in C57BL/6 mice fed an atherogenic diet. Atherosclerosis, 178:2659, 2005.

Bankova, V. S.; de Castro, S. L. \& Marcucci, M. C. Propolis: recent advances in chemistry and plant origin. Apidologie, 31:3-15, 2000.

Banskota, A. H.; Tezuka, Y. \& Kadota, S. Recent progress in pharmacological research of propolis. Phytother Res., 15:561-71, 2001.

Barham, D. \& Trinder, P. An improved colour reagent for the determination of blood glucose by the oxidase system. Analyst, 97:142-5, 1972.

Basnet, P.; Matsushige, K.; Hase, K.; Kadota, S. \& Namba, T. Four di-O-caffeoyl quinic acid derivatives from propolis. Potent hepatoprotective activity in experimental liver injury models. Biol. Pharm. Bull., 19:147984, 1996.

Bergmeyer, H.V.; Bowers, G.N.; Horder, M. \& Mas, A.W. Optimization of methods for aspartate aminotransferase and alanine amino transferase Clin. Chim. Acta, 24:5873, 1978.

Bhadauria, M.; Nirala, S. K. \& Shukla, S. Durationdependent hepatoprotective effects of propolis extract against carbon tetrachloride-induced acute liver damage in rats. Adv. Ther., 24:1136-45, 2007.

Bhadauria, M.; Nirala, S. K. \& Shukla, S. Multiple treatment of propolis extract ameliorates carbon tetrachloride induced liver injury in rats. Food Chem. Toxicol., 46: 2703-12, 2008.
Bujanda, L.; Hijona, E.; Larzabal, M.; Beraza, M.; Aldazabal, P.; Garcia-Urkia, N.; Sarasqueta, C.; Cosme, A.; Irastorza, B.; Gonzalez, A. \& Arenas, J. I., Jr. Resveratrol inhibits nonalcoholic fatty liver disease in rats. BMC Gastroenterol., 8:40, 2008.

Burdock, G. A. Review of the biological properties and toxicity of bee propolis (propolis). Food Chem. Toxicol., 36:347-63, 1998.

Chen, C. S.; Wu, C. H.; Lai, Y. C.; Lee, W. S.; Chen, H. M.; Chen, R. J.; Chen, L. C.; Ho, Y. S. \& Wang, Y. J. NF-kappaB-activated tissue transglutaminase is involved in ethanol-induced hepatic injury and the possible role of propolis in preventing fibrogenesis. Toxicology, 246: 148-57, 2008.

Desai, M. S.; Mariscalco, M. M.; Tawil, A.; Vallejo, J. G. \& Smith, C. W. Atherogenic diet-induced hepatitis is partially dependent on murine TLR4. J. Leukoc. Biol., 83:1336-44, 2008.

Flavio, L.; Lorenzo, A.; Serena, O.; Rocco, O. \& Francesco, A. Antioxidant supplements for non-alcoholic fatty liver disease and/or steatohepatitis. Cochrane Database Syst. Rev., 24:2007.

Fossati, P. \& Medicci, R. Abstract Book. International Symposium on Cholesterol Control and Cardiovascular Diseases: Prevention and Therapy. Milan Italy, 1987. Apud: Bayer Corporation, Diagnostic Division, Tarrytown, N.Y., Cholesterol-Fast color.

Fossati, P. \& Prencipe, L. Serum triglycerides determined colorimetrically with an enzyme of low-density lipoprotein cholesterol in plasma without use of the preparative ultracentrifuge. Clin. Chem., 28:2077-80, 1982.

Getz, G. S. \& Reardon, C. A. Diet and Murine Atherosclerosis. Arterioscler. Thromb. Vasc. Biol., 26:242-9, 2006.

Giurgea, R.; Rusu, M. A.; Coprean, D.; Popescu, H. \& Polinicencu, C. Biochemical effects of standardized propolis extract (SPE) and of silymarin on the liver of ethyl alcohol intoxicated rats. Agressologie, 28:831-2, 1987.

Hegazi, A. G.; Abd El Hady, F. K. \& Abd Allah, F. A. Chemical composition and antimicrobial activity of European propolis. Z. Naturforsch C., 55:70-5, 2000. 
Herrera, C. L.; Alvear, M.; Barrientos, L.; Montenegro, G. \& Salazar, L. A. The antifungal effect of six commercial extracts of chilean propolis on Candida spp. Cienc. Inv. Agr., 2010 (in press).

Ichi, I.; Hori, H.; Takashima, Y.; Adachi, N.; Kataoka, R.; Okihara, K.; Hashimoto, K. \& Kojo, S. The beneficial effect of propolis on fat accumulation and lipid metabolism in rats fed a high-fat diet. J. Food Sci., 74: H127-31, 2009.

Kleiner, D. E.; Brunt, E. M.; Van Natta, M.; Behling, C.; Contos, M. J.; Cummings, O. W.; Ferrell, L. D.; Liu, Y. C.; Torbenson, M. S.; Unalp-Arida, A.; Yeh, M.; McCullough, A. J. \& Sanyal, A. J. Design and validation of a histological scoring system for nonalcoholic fatty liver disease. Hepatology, 41:131321, 2005.

Koya-Miyata, S.; Arai, N.; Mizote, A.; Taniguchi, Y.; Ushio, S.; Iwaki, K. \& Fukuda, S. Propolis prevents diet-induced hyperlipidemia and mitigates weight gain in diet-induced obesity in mice. Biol. Pharm. Bull., 32:2022-8, 2009.

Kujumgiev, A.; Tsvetkova, I.; Serkedjieva, Y.; Bankova, V.; Christov, R. \& Popov, S. Antibacterial, antifungal and antiviral activity of propolis of different geographic origin. J. Ethnopharmacol., 64:235-40, 1999.

Larter, C. Z. \& Yeh, M. M. Animal models of NASH: getting both pathology and metabolic context right. $J$. Gastroenterol. Hepatol., 23:1635-48, 2008.

Lin, S. C.; Chung, C. Y.; Chiang, C. L. \& Hsu, S. H. The influence of propolis ethanol extract on liver microsomal enzymes and glutathione after chronic alcohol administration. Am. J. Chin. Med., 27:83-93, 1999.

Lin, S. C.; Lin, Y. H.; Chen, C. F.; Chung, C. Y. \& Hsu, S. $\mathrm{H}$. The hepatoprotective and therapeutic effects of propolis ethanol extract on chronic alcohol-induced liver injuries. Am. J. Chin. Med., 25:325-32, 1997.

Machado, M. \& Cortez-Pinto, H. Non-alcoholic steatohepatitis and metabolic syndrome. Curr. Opin. Clin. Nutr. Metab. Care, 9:637-42, 2006.

Marcucci, M. C.; Ferreres, F.; Custodio, A. R.; Ferreira, M. M.; Bankova, V. S.; Garcia-Viguera, C. \& Bretz, W. A. Evaluation of phenolic compounds in Brazilian propolis from different geographic regions. $Z$. Naturforsch C. 55:76-81, 2000.
Matsuzawa, N.; Takamura, T.; Kurita, S.; Misu, H.; Ota, T.; Ando, H.; Yokoyama, M.; Honda, M.; Zen, Y.; Nakanuma, Y.; Miyamoto, K. \& Kaneko, S. Lipidinduced oxidative stress causes steatohepatitis in mice fed an atherogenic diet. Hepatology, 46:1392-403, 2007.

Merino, N.; Gonzalez, R.; Gonzalez, A. \& Remirez, D. Histopathological evaluation on the effect of red propolis on liver damage induced by $\mathrm{CCl} 4$ in rats. Arch. Med. Res., 27:285-9, 1996.

Montenegro, G.; Mujica, A. M.; Peña, R. C.; Gomez, M.; Serey, I. \& Timmermann, B. N. Similitude pattern and botanical origin of the Chilean propolis. Phyton., 73:145-54, 2004.

Nakajima, Y.; Tsuruma, K.; Shimazawa, M.; Mishima, S. \& Hara, H. Comparison of bee products based on assays of antioxidant capacities. BMC Complement. Altern. Med., 9:4, 2009.

Nirala, S. K. \& Bhadauria, M. Propolis reverses acetaminophen induced acute hepatorenal alterations: a biochemical and histopathological approach. Arch. Pharm. Res., 31:451-61, 2008.

Paigen, B.; Morrow, A.; Holmes, P. A.; Mitchell, D. \& Williams, R. A. Quantitative assessment of atherosclerotic lesions in mice. Atherosclerosis, 68:231-40, 1987.

Peña, R. C. Estandarización en propóleos: antecedentes químicos y biológicos. Cienc Inv Agr. 35: 17-26, 2008.

Sathiavelu, J.; Senapathy, G. J.; Devaraj, R. \& Namasivayam, N. Hepatoprotective effect of chrysin on prooxidant-antioxidant status during ethanolinduced toxicity in female albino rats. J. Pharm. Pharmacol., 61:809-17, 2009.

Schreuder, T. C.; Verwer, B. J.; van Nieuwkerk, C. M. \& Mulder, C. J. Nonalcoholic fatty liver disease: an overview of current insights in pathogenesis, diagnosis and treatment. World J. Gastroenterol., 14:247486, 2008.

Seo, K. W.; Park, M.; Song, Y. J.; Kim, S. J. \& Yoon, K. R. The protective effects of Propolis on hepatic injury and its mechanism. Phytother Res., 17:250-3, 2003.

Sforcin, J. M. Propolis and the immune system: a review. J. Ethnopharmacol., 113:1-14, 2007. 
HERRERA, C. L.; FRITZ, O.; MONTENEGRO, G., ALVEAR, M.; DEL SOL, M. \& SALAZAR, L. A. El propóleos reduce la esteatosis hepática inducida por dieta en ratones. Int. J. Morphol., 28(1):75-84, 2010

Shang, J.; Chen, L. L. \& Xiao, F. X. [Resveratrol improves high-fat induced nonalcoholic fatty liver in rats]. Zhonghua Gan. Zang Bing Za. Zhi., 16:616-9, 2008.

Stokes, K. Y. Microvascular Responses to Hypercholesterolemia: The Interactions Between Innate and Adaptive Immune Responses. Antioxidants \& Redox Signaling, 8:1141-51, 2006.

Velikova, M.; Bankova, V.; Marcucci, M. C.; Tsvetkova, I. \& Kujumgiev, A. Chemical composition and biological activity of propolis from Brazilian meliponinae. $Z$. Naturforsch C., 55:785-9, 2000.

Vergnes, L.; Phan, J.; Strauss, M.; Tafuri, S. \& Reue, K. Cholesterol and cholate components of an atherogenic diet induce distinct stages of hepatic inflammatory gene expression. J. Biol. Chem., 278:42774-84, 2003.

Wittenburg, H.; Lyons, M. A.; Paigen, B. \& Carey, M. C. Mapping cholesterol gallstone susceptibility (Lith) genes in inbred mice. Dig Liver Dis. 35 Suppl 3: S2-7, 2003.

Yan, E.; Durazo, F.; Tong, M. \& Hong, K. Nonalcoholic fatty liver disease: pathogenesis, identification, progression, and management. Nutr. Rev., 65:376-84, 2007.
Dirección para Correspondencia:

Prof. Dr. Luis Antonio Salazar

Departamento de Ciencias Básicas,

Facultad de Medicina

Universidad de La Frontera

Av. Francisco Salazar 01145

Casilla 54-D

Temuco, Chile

Email: Isalazar@ufro.cl

Recibido : 22-12-2009

Aprobado:15-01-2010 